\title{
TRANSPORT PROCESSES IN THE PLASMA EDGE
}

\author{
Bernhard Unterberg \\ Institut für Energieforschung - Plasmaphysik, Forschungszentrum Jülich GmbH, Association EURATOM-FZ Jülich \\ D-52425 Jülich, Germany, phone: +49 246161 4803, email: B.Unterberg@fz-juelich.de
}

\begin{abstract}
Basic properties of the plasma edge in magnetically confined fusion plasmas are summarized. Starting from the magnetic topology of tokamaks we describe the transport of the scrape-off layer including drifts, the transition to high recycling and detached plasma regimes typical for divertors and the consequences of the electrostatic Debye sheath in front of the plasma facing components. The transport of the fuel neutrals (hydrogen atoms and molecules) is introduced.
\end{abstract}

\section{INTRODUCTION}

Processes at the edge plasma in general and plasma-wall interaction in particular play a crucial role for achieving a steady state burning fusion plasma. The first wall has to withstand and exhaust the $\alpha$ - particle heating power and the helium-ash must be removed (pumped) from the plasma. Wall erosion affects the lifetime of wall elements and releases impurities into the plasma, which then cause fuel dilution and energy loss due to radiation from the plasma centre. Moreover, also global confinement properties can be affected by edge processes. Therefore, understanding these processes and controlling the edge plasma by appropriate means is an important field of research (cf. also [1] for an introduction to the field of plasma- wall interaction).

The plasma in a tokamak or stellarator represents an open system. The wall is a perfect plasma sink and owing to the finite confinement times (energy and particle confinement times $\tau_{E}$ and $\tau_{P}$ ) the plasma has to be renewed continuously. The energy content $\mathrm{E}$ is sustained by heating, $E=P_{\text {heat }} * \tau_{E}$, with the heating power $P_{\text {heat }}$. The number $N$ of particles in the plasma is sustained by a permanent flow of $\mathrm{D} / \mathrm{T}$ gas from the wall elements into the plasma, $N=\Gamma * \tau_{p}$. The alpha particles with a power density of $P_{\alpha}=0.15 \mathrm{MW} / \mathrm{m}^{3}$ $\left(\mathrm{T}=10 \mathrm{keV}, n=10^{20} \mathrm{~m}^{-3}\right.$ ) lead to an average wall load of some $100 \mathrm{~kW} / \mathrm{m}^{2}$, if we take into account a plasma volume of roughly $1000 \mathrm{~m}^{3}$ for self- sustained burn - a

\footnotetext{
*Partner in the Trilateral Euregio Cluster
}

moderate value. However, energy exhaust becomes a problem, because the magnetic field directs the convected heat load on rather small wall areas. This can lead to peak loads which could damage the wall.

Another important issue of edge physics is impurity generation and impurity exhaust. Any impurities in the plasma centre lead to fuel dilution reducing the fusion power. Their concentration has to stay below a certain level. E.g. the concentration of the unavoidable helium, which is generated at a rate of $R_{H e}=10^{18} \mathrm{~s}^{-1} \mathrm{~m}^{-3}$, should not exceed significantly a value of about $10 \%$. This condition is fulfilled when the characteristic time $\tau_{p, H e}^{*}$ for helium removal is sufficiently low [9], [10]. The experimental values found for $\tau_{p, H e}^{*}$ are much larger (factor 10 or more) than the global confinement time of helium demonstrating that helium is recycling at the wall more than 10 times before it is removed by the pumps. The presence of other impurities in the plasma depends on the choice of wall materials, erosion processes and edge plasma properties, like temperature, density and particle transport (for a further description of impurity transport and radiation processes $\mathrm{cf}$. [11]).

No unique definition exists for the term "plasma edge" or "plasma boundary". An important part of the edge plasma is the scrape-off layer (SOL) which is that region of the plasma where the magnetic field lines intersect wall elements. But significant processes occur also inside the confined plasma, like neutral particle penetration, ionization, charge exchange or impurity line radiation. These atomic processes have an impact on the properties of both, the edge plasma and the core plasma.

In this lecture plasma edge physics is introduced comprising the SOL as well as part of the confined plasma. The relevant processes are discussed following the transport cycle of the particles beginning with the boundary conditions which are given by the magnetic topology. After an illustration of the basic SOL properties, we discuss the penetration of neutrals into the plasma. Then a general description of the parallel transport in the SOL is given. Next, we introduce the Debye sheath in front of the plasma facing components, 
before we finally discuss the properties of the simple limiter SOL and the complex divertor SOL. Overall, the physical processes in the plasma edge have important consequences for the interaction between plasma and wall and the resulting recycling and erosion mechanisms. This subject is discussed in a separate lecture $[2]$.

An excellent introduction into the physics of the plasma boundary can be found in the book of P.C. Stangeby "The Plasma Boundary of Magnetic Fusion Devices" [3]. Further monographs used for this overview have been published by A.V. Nedospasov and M.Z. Tokar [4] and R. Schneider [5], a review article on experimental divertor physics by C.S. Pitcher and P.C. Stangeby is published in [6].

\section{MAGNETIC TOPOLOGY}

Wall elements which intersect the magnetic field serve as a perfect plasma sink and impose a flow directed along the field lines. The flux tubes generated at each wall element are filled with plasma by perpendicular transport (diffusion, drifts). This property helps to build up a particle density sufficient for helium exhaust. On the other hand the concentration of plasma flow on small areas is less beneficial for power exhaust, since a uniform plasma flow to the whole wall would avoid peak heat loads. The very details of particle and heat load on the wall are determined by the magnetic topology and the geometry of the plasma facing components. We have to distinguish two different concepts: divertor and limiter. The poloidal divertor shown in Fig. 1 is used in the performance oriented tokamak devices (JET, JT60U, DIII-D, ASDEX-U) and is the preferred concept for the next step device. The simpler (and cheaper) limiter is explored e.g. in Tore Supra and TEXTOR (toroidal belt limiter), in particular, with respect to steady state technology, plasma-wall interaction and new concepts with ergodic boundaries. The subject of ergodic divertor physics is discussed in [7], for a review on divertor physics in stellarators the reader is referred to [8].

The projection of the flux tubes on the surface of the limiter/divertor plate is determined by two angles: the tilting angle $\alpha$ between toroidal and poloidal direction depends on the rotational transform (safety factor) $q$; the angle $\Psi$ in the poloidal plane between the magnetic field and the surface normal is given by the limiter shape or the orientation of the divertor plates. In torus geometry $\alpha$ varies along the poloidal coordinate depending on the aspect ratio and the plasma pressure.

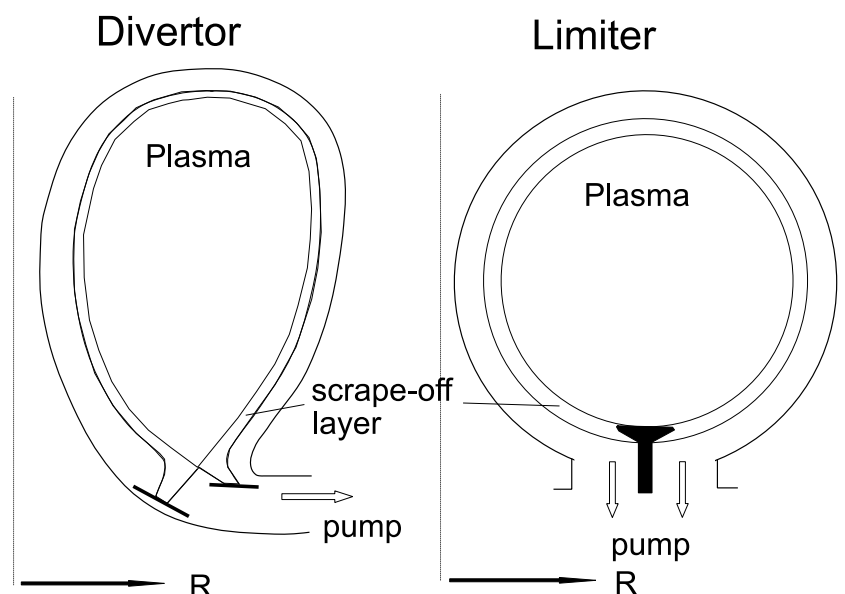

Figure 1: Poloidal divertor and toroidal limiter concept shown in the poloidal plane of a tokamak

\section{BASIC CHARACTERISTICS OF THE SOL}

Both concepts shown in fig. 1 are toroidally symmetric. This allows to discuss the main features by reducing the transport inside the SOL to a 2-dimensional problem: flow along the field line (coordinate $z$ ) and diffusion in radial direction (coordinate $x$ ) as shown in fig. 2. As we will see below, the radial extension of the SOL is small with respect to the minor radius of the plasma $(\lambda \ll a)$, so that we can apply a plane geometry and straighten out the SOL as indicated in fig. 2, thereby neglecting toroidal effects.

The SOL begins at the last closed flux surface (LCFS). After the initiation of the plasma discharge and on a time scale of $\mu s$, electrons will rush ahead the ions as a consequence of their higher mobility and charge up the solid negatively. A thin sheath will form to shield the electrostatic potential with a characteristic length given by the so called Debye length

$$
\lambda_{D}=\sqrt{\frac{\epsilon_{0} k T_{e}}{n_{e} e^{2}}}
$$

For $T_{e}=20 \mathrm{eV}$ and $n_{e}=10^{19} \mathrm{~m}^{-3}$ we get $\lambda_{D}=$ $10^{-5} \mathrm{~m}$, which reflects the fact that the plasma maintains quasi- neutrality very well. Further properties of this sheath will be discussed in section VI.

However, the shielding is imperfect because of the thermal motion of the plasma particles and a small electric field penetrates the plasma (pre-sheath) which accelerates the ions towards the target. The electrons feel a corresponding retarding field. The plasma fluid as a whole is quasi- neutral, because of the plasma sink at the end of the field lines a pressure gradient develops and a symmetric flow towards both ends is driven. At the symmetry plane the parallel flow velocity $v_{\|}$and the 
a)

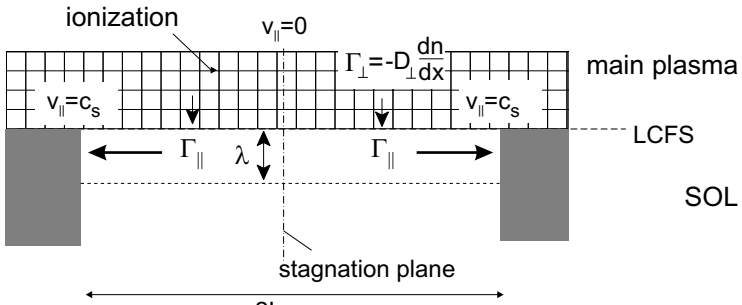

b)

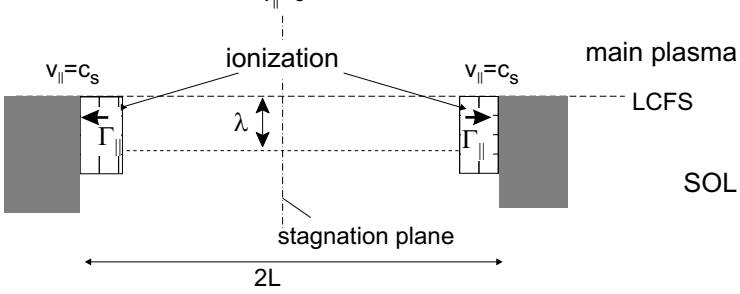

Figure 2: 2-d model of the SOL, a) simple SOL, b) complex SOL

parallel flux density $\Gamma_{\|}$must be zero (stagnation plane) and at both ends the flow velocity reaches sound speed, $v_{\|}=c_{s}$ (so called the "Bohm criterion").

At the material surfaces ions and electrons recombine and are released back into the plasma as neutrals, will be ionized by impact of electrons and form the plasma source to drive again plasma flow back to the material surfaces. This process which has a very short time scale compared to the duration of a plasma discharge (e.g. in TEXTOR several tens of ms compared to $10 \mathrm{~s}$ ) is called recycling. The recycling process is strongly influenced by the fact how the neutrals are released into the plasma and where they get ionized. Some characteristics of the transport of neutrals in the plasma edge are summarized in section IV. Much more details, in particular on techniques how to prescribe the neutrals in realistic geometry are given in [9]. Two different situations can be distinguished with respect to the particle sources in the SOL. Under the simplified assumption that there are no ionization sources in the volume of the SOL but the sources are located inside the confined volume, the SOL is solely filled by a crossfield diffusion with a flux density $\Gamma=-D_{\perp} \partial n / \partial x$. This situation is generally named the "simple SOL", cf. fig. 2a). On the other hand, if all ionization sources are located inside the SOL, no perpendicular diffusion into the SOL develops, cf. fig. 2b). While the first situation is usually realized in limiter tokamaks and in divertor tokamaks with low plasma densities, the second situation can only be realized in divertor tokamaks at high plasma densities and is usually called the "complex SOL" for reasons which will become clear in the following.

Throughout this overview we will describe the edge plasma in the fluid picture with equations for the average quantities rather than with a full kinetic analysis. Strictly, the requirement for such a procedure is that the self- collisional mean free pathes of electrons and ions $\lambda_{e e} \approx \lambda_{i i} \approx 10^{16} T^{2} / n$ (with $T$ in $\mathrm{eV}$ and $n$ in $m^{-3}$ yielding $\lambda_{e e, i i}$ in $\mathrm{m}$ ) are smaller than the extension of the plasma along the magnetic field (connection length L). In the medium size limiter tokamak TEXTOR with typical SOL parameters $L \approx 20 \mathrm{~m}$, $n=1-10 \cdot 10^{18} m^{-3}$ and $T=10-100 \mathrm{eV}$ the mean free path is $0.1 \mathrm{~m}$ (high density/ low temperature case) up to $100 \mathrm{~m}$ (low density/ high temperature case) the situation can be marginal at SOL low densities.

With simple estimates we may now characterize some basic features of such a SOL as the SOL thickness and the radial density variation, assuming a simple SOL with perpendicular diffusion as particle source for the SOL. For this first estimate we may relate the length of the flux tube (connection length $2 L$, where $L=\pi q_{a} R$ in a toroidal limiter / poloidal divertor configuration with $q_{a}$ the safety factor at the edge and $R$ the major radius) and the SOL thickness $\lambda$ to the average transport velocities $v_{\|}$and $v_{\perp}$ according to

$$
\frac{v_{\perp}}{v_{\|}}=\frac{\lambda}{L} .
$$

For the average velocities we take $v_{\|}=0.5 c_{s}$ and $v_{\perp} n=D_{\perp} \partial n / \partial x$. With the characteristic length $\lambda=$ $(1 / n) \partial n / \partial x$ we obtain for Eq. 2

$$
\frac{D_{\perp} / \lambda}{0.5 c_{s}}=\frac{\lambda}{L}
$$

From this relation we get the well known expression for the SOL thickness $\lambda$ (i.e. the density decay length)

$$
\lambda=\sqrt{\frac{D_{\perp} L}{0.5 c_{s}}}
$$

With typical values for an edge plasma $D_{\perp}=$ $1 \mathrm{~m}^{2} / \mathrm{s}, T=50 \mathrm{eV}$, and $\mathrm{L}=10 \mathrm{~m}$ we obtain $\lambda=30 \mathrm{~mm}$. This is a remarkably small value compared to the dimensions of a fusion reactor. As a consequence, the surface area wetted by the plasma reduces by roughly two orders of magnitude with respect to the total wall area, leading to unacceptable high heat loads. We will see later that this simple calculation even overestimates the SOL thickness, in particular close to the plasma facing components.

The radial variation $f(x)$ of density inside the SOL can be derived from a simple 1d-calculation based on the conservation of mass along the flow channel $\mathrm{z}$

$$
\frac{\partial}{\partial x} D_{\perp} \frac{\partial n}{\partial x}=\frac{\partial}{\partial z}\left(n v_{\|}\right)
$$


Assuming in a first step $D_{\perp}=$ const and $\partial n / \partial x=$ const. along $\mathrm{z}$ as well as a constant r.h.s of Eq. 5 represented by $\partial / \partial z\left(n v_{\|}\right)=n / \tau_{\|}$with a characteristic particle residence time in the SOL given by $\tau_{\|}$(parallel transport to the target is the only plasma sink, no particle sources caused by ionization of neutrals inside the SOL are considered) we obtain the solution of Eq. 5

$$
n(x)=n(0) \exp \left(-x / \sqrt{D_{\perp} \tau_{\|}}\right)
$$

The density shows an exponential decay inside the SOL with a characteristic length $\lambda=\sqrt{D_{\perp} \tau_{\|}}$as given by Eq. $4, n(0)$ denotes the density at the LCFS. Here, the typical time scale of parallel transport to the targets $\tau_{\|}$is of the order of ms. However, one has to be careful when using these simple expressions, as particle sources inside the SOL and drifts will alter the result as discussed later on.

\section{NEUTRAL PARTICLE TRANSPORT}

Hydrogen (deuterium or tritium) and impurity neutrals are released from the plasma facing components and penetrate into the edge plasma. Owing to the different release mechanisms as discussed in [2] we observe also different particle velocities. This has an important impact on the edge plasma.

Hydrogen may be released as a molecule $H_{2}$ or an atom $H_{0}$. It has been found that in the recycling process the probability for molecule formation depends on the surface temperature, which determines the residence time in the surface. At low temperatures mainly molecules are desorbed while above about $T_{s}=1200 \mathrm{~K}$ the majority of particles is released as atoms [19] [20].

Some processes involved with the penetration of $\mathrm{H}_{2}$ are illustrated in Fig. 3 . Because of electron impact the molecule dissociates. Various dissociation channels compete, with cross sections depending on $T_{e}$. Some of them are given in table 1 together with the rate coefficients for $T_{e}=50 \mathrm{eV}$.

The reaction no.1, also illustrated in Fig. 3, is only dominant at or below $T_{e}=10 \mathrm{eV}$, whereas at higher $T_{e}$ the molecule is first ionized and then dissociated (reactions no.3 and no.4), as is obvious from the rate coefficients $\langle\sigma v\rangle_{\text {dis }}[21]$. The atoms resulting from the dissociation of molecules in ground state gain energies in the range of $2.2 \mathrm{eV}$. Surprisingly, average energies significantly lower than these $(0.5 \mathrm{eV})$ have been measured in the vicinity of a limiter [22] [23]. It is assumed that this is caused by vibrationally excited molecules.

The probability that an atom has at least one charge exchange $(\mathrm{CX})$ reaction before it is ionized is rather high because the rate coefficients for ionization

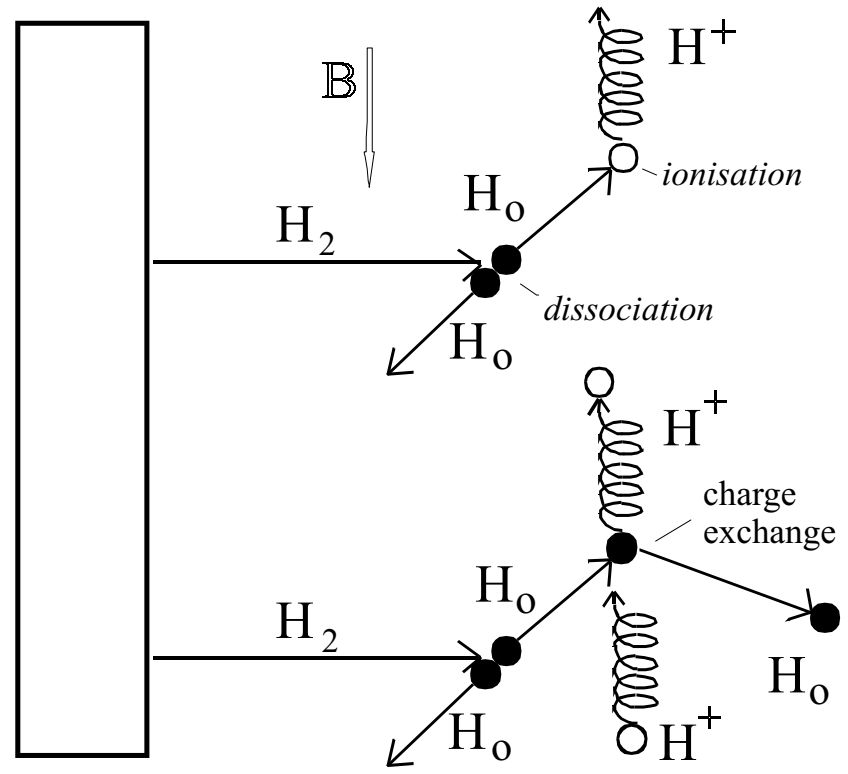

Figure 3: Molecule dissociation and charge exchange processes at the plasma boundary (solid circles: atoms, open circles: ions)

\begin{tabular}{|c|l|r|}
\hline no. & dissociation reaction & $\langle\sigma v\rangle_{\text {dis }} / m^{3} / s$ \\
\hline 1 & $H_{2} \rightarrow H_{0}+H_{0}$ & $6 \cdot 10^{-15}$ \\
2 & $H_{2} \rightarrow H_{0}+H^{+}$ & $2 \cdot 10^{-15}$ \\
3 & $H_{2} \rightarrow H_{2}^{+}$ & $4 \cdot 10^{-14}$ \\
4 & $H_{2}^{+} \rightarrow H_{0}+H^{+}$ & $3 \cdot 10^{-13}$ \\
5 & $H_{2}^{+} \rightarrow H^{+}+H^{+}$ & $6 \cdot 10^{-15}$ \\
\hline
\end{tabular}

Table 1: Dissociation reactions of hydrogen molecules and molecular ions[21].

$\langle\sigma v\rangle_{i}$ and $\mathrm{CX}\langle\sigma v\rangle_{C X}$ are similar as is shown in the table 2 [21].

Using the atomic and molecular data the transport of neutral particles can be modelled with a rather high accuracy even for complicated 3d-geometries [24] [9].

The velocity of impurity atoms also depends on their release mechanisms. The fastest particles are reflected impurities (impurity ions from the plasma, neutralized and re-emitted). Among the erosion mechanisms, sputtering generates particles in the range of $5 \mathrm{eV}$. Atoms coming from molecules gain their velocity from the dissociation energy. Sublimated or evap-

\begin{tabular}{|c|r|r|l|}
\hline$T_{e}=T_{i}$ & 10 & 100 & $\mathrm{eV}$ \\
$\langle\sigma v\rangle_{i}$ & $7 \cdot 10^{-15}$ & $3 \cdot 10^{-14}$ & $\mathrm{~m}^{3} \mathrm{~s}^{-1}$ \\
$\langle\sigma v\rangle_{C X}$ & $2 \cdot 10^{-14}$ & $5 \cdot 10^{-14}$ & $\mathrm{~m}^{3} \mathrm{~s}^{-1}$ \\
\hline
\end{tabular}

Table 2: Rate coefficients for ionization and charge exchange [21]. 
orated atoms have only thermal energy, thus represent the slowest particles with the least impact on the edge plasma (cf. discussion in [2]).

Recombination processes are generally not important unless the plasma is very cold as in detached divertors, since in most cases the recombination times of ions are much longer than the average residence time of the particles in the plasma.

The ionization time of an atom can be calculated from the rate coefficient for ionization $\langle\sigma v\rangle_{i}\left(T_{e}\right)$ and the local electron density $n_{e}$. The time derivative of the neutral density $n_{0}$ owing to ionization is then given by

$$
\frac{\partial n_{0}}{\partial t}=-n_{e} n_{0}<\sigma v>_{i}
$$

leading to an exponential decay of the atom density

$$
n_{0}(t)=n_{0}(t=0) \exp \left(-\frac{t}{\tau_{i}}\right) .
$$

The ionization time $\tau_{i}$ is given by

$$
\tau_{i}=\frac{1}{n_{e}<\sigma v>_{i}}
$$

The penetration of neutral particles into a homogeneous plasma having a velocity $v_{0}$ is given by the ionization length

$$
\lambda_{i}=\frac{v_{0}}{n_{e}<\sigma v>_{i}}
$$

In the presence of a radial profile of both the electron density and temperature the description of the ionization length can be generalized to

$$
\int_{0}^{\lambda_{i}} \frac{n_{e}(r)<\sigma v>_{i}\left(T_{e}(r)\right)}{v_{0}} d r=1
$$

We can define the ion source distribution $Q(r)$, which is given under steady- state conditions as

$$
Q(r)=-\frac{\partial \Gamma_{0}}{\partial r}=n_{0}(r) n_{e}(r)<\sigma v>_{i}\left(T_{e}(r)\right)
$$

Here, $\Gamma_{0}=n_{0} v_{0}$ is the neutral flux density under the simplifying assumption of a mono-energetic neutral velocity distribution. The ionization length $\lambda_{i}$ can be used to characterize the radial extent of the ion source distribution. As a consequence the impact of neutrals on the edge plasma is characterized both by their velocities as determined by the specific release mechanism and the edge plasma parameters $n_{e}$ and $T_{e}$.

As stated before, for hydrogen atoms charge exchange processes are important in addition. This gives rise to a diffusion process of the atoms. The penetration depth is given by the geometric mean of the ionization length $\lambda_{i}$ as given by Eq. 10 and the mean free path for the CX process $\lambda_{C X}$ [25] using for both (!) the thermal ion velocity $v_{t h}=\sqrt{k T_{i} / m_{i}}$

$$
\lambda_{p e n}=\frac{v_{t h}}{n_{e} \sqrt{<\sigma v>_{C X}<\sigma v>_{i}}} .
$$

\section{GENERAL DESCRIPTION OF PARALLEL TRANSPORT IN THE SOL}

We start our general description of SOL transport parallel to the magnetic field (z direction) in the fluid picture with the conservation equations for particles, momentum and energy under steady- stated conditions (no partial time derivatives). The extension to $2 \mathrm{D}$ transport (parallel - radial) can be made by including "effective" cross field sources (cf. [3], chapter 13, and [4], section 1.1). We consider two species (electrons $e$ and singly charged plasma ions $i$ ) under the assumption of quasi- neutrality $\left(n_{e}=n_{i}=n\right)$.

$$
\frac{\partial}{\partial z}\left(n_{i, e} v_{\| i, e}\right)=S_{p}
$$

Here $n$ denotes the particle density, $v_{\|}$the parallel fluid velocity and $S_{p}$ the particle source which can be related to particle transport into the SOL, to ionization or recombination processes (the latter forming a particle sink in the SOL volume).

Next we introduce the momentum equations for plasma ions of mass $m_{i}$ in its conservative form

$$
\frac{\partial}{\partial z}\left(m_{i} n v_{i, \|}^{2}+p_{i}\right)=e n E+R_{i e}+R_{n} .
$$

Here $p_{i}=k T_{i} n$ is the ion pressure with $T_{i}$ the ion temperature, $k$ the Boltzmann constant, $e$ the elementary charge, $E$ the parallel electric field, $R_{i e}$ the friction force term owing to collisions with electrons and $R_{n}$ the friction force term owing to collisions with neutrals. Both friction terms have two contributions,

$$
R_{i e}=m_{e}\left(v_{e}-v_{i}\right) \nu_{e i} n+0.71 n \frac{\partial k T_{e}}{\partial z}
$$

with $m_{e}$ the electron mass, $\nu_{e i}$ the electron ion collision frequency and $T_{e}$ the electron temperature, and

$$
R_{n}=-m_{i}\left(v_{i}-v_{n}\right)<\sigma v>_{C X} n_{n} n+m_{i} v_{n} S_{p}
$$

with $v_{n}$ the velocity which with the neutrals with a Maxwellian distribution are drifting, $\langle\sigma v\rangle_{C X}$ the rate coefficient for charge exchange between ions and neutrals and $n_{n}$ the neutral density.

For electrons the inertia term $\frac{\partial}{\partial z}\left(m_{e} n v_{e, \|}^{2}\right)$ can be neglected as well as electron momentum sources because of the small electron mass, and we are left with 


$$
\frac{\partial}{\partial z} p_{e}+e n E=-m_{e}\left(v_{e}-v_{i}\right) \nu_{e i} n-0.71 n \frac{\partial k T_{e}}{\partial z}
$$

If we solve equation 18 for the parallel electric field, we obtain Ohm's law as

$$
E=\frac{j_{\|}}{\sigma_{\|}}-\frac{0.71}{e} \frac{\partial k T_{e}}{\partial z}-\frac{1}{e n} \frac{\partial p_{e}}{\partial z}
$$

where the parallel current density has been defined as $j_{\|}=e n\left(v_{i}-v_{e}\right)$ and the parallel electric conductivity as derived assuming balance between electric and e-i friction force as $\sigma_{\|}=e^{2} n /\left(m_{e} \nu_{e i}\right)$. In most cases one assumes local ambipolarity, $j_{\|}=0$ such that for a deuterium plasma $v_{e}=v_{i}=v$. Otherwise, a current continuity equation is needed e.g. to describe cases where external currents are driven in biasing experiments (cf. also the discussion in [3], chapter 17).

Next we proceed to the energy conservation equation for ions, which is given as

$$
\begin{aligned}
\frac{\partial q_{\| i}}{\partial z} & =\frac{\partial q_{\| i, \text { conv }}}{\partial z}+\frac{\partial q_{\| i, \text { cond }}}{\partial z} \\
& =\frac{\partial}{\partial z}\left[\left(\frac{5}{2} T_{i}+\frac{1}{2} m_{i} v_{i}^{2}\right) n v_{i}-\kappa_{0 i} T_{i}^{5 / 2} \frac{\partial T_{i}}{\partial z}\right] \\
& =\text { env }_{i} E++Q_{\text {in }}-Q_{\text {eq }},
\end{aligned}
$$

where we have decomposed the parallel heat flux into its convective and conductive part. The heat conduction coefficient $K=\kappa_{o i} T_{i}^{5 / 2}$ has a very strong temperature dependence, for ions we have for deuterium ions $\kappa_{o i}=60$ to get with $T_{i}$ in $e V$ and $\partial T_{i} / \partial z$ in $e V m^{-1}$ the conductive heat flux in $W m^{-2}$. The term $Q_{\text {in }}$ denotes the energy exchange between ions and neutrals during charge exchange processes, $Q_{e q}$ is the energy transport from ions to electrons in Coulomb collisions.

For electrons we get accordingly

$$
\begin{aligned}
\frac{\partial q_{\| e}}{\partial z} & =\frac{\partial q_{\| e, \text { conv }}}{\partial z}+\frac{\partial q_{\| e, \text { cond }}}{\partial z} \\
& =\frac{\partial}{\partial z}\left[\frac{5}{2} T_{e} n v_{e}-\kappa_{0 e} T_{e}^{5 / 2} \frac{\partial T_{e}}{\partial z}\right] \\
& =- \text { env }_{e} E+Q_{r}-Q_{e i}+Q_{e q}
\end{aligned}
$$

Again we have omitted the inertia term in the convective flux, $Q_{r}$ denotes the Joule heating term (not present if $\left.j_{\|}=0\right), Q_{e i}$ is the energy loss of electrons because of inelastic collisions which ionize or excite neutrals. The heat conduction by electrons is substantially larger than that of ions, $\kappa_{o e}=2000$, because of the $m^{-1 / 2}$ dependence of the heat conductivity.
Now we are still missing boundary conditions for our fluid equations $6,15,18,20$ and 21. At the stagnation plane we request from symmetry considerations

$$
v_{\|}=\frac{\partial n}{\partial z}=\frac{\partial T_{i}}{\partial z}=\frac{\partial T_{e}}{\partial z}=0 .
$$

The boundary conditions at the limiter and target plate are defined by the existence of the Debye sheath mentioned before, which is the subject of the next section.

\section{THE SHEATH}

Within the Debye sheath quasi - neutrality is no longer fulfilled and the electrostatic potential is given by Poisson's equation

$$
\frac{\partial^{2} V}{\partial z}=-\frac{e}{\varepsilon_{0}}\left(n_{i}-n_{e}\right)
$$

In this potential the electrons can be described by a Boltzmann equation

$$
n_{e}(z)=n_{s e} \exp \left(e\left(V-V_{s e}\right) / k T_{e}\right)
$$

where $n_{s e, e}=n_{s e, i}=n_{s e}$ is the density at the sheath entrance. This potential distribution constitutes a hill for the electrons $(V<0)$, as the limiter or divertor surface has initially been charged negatively by the electrons. At the sheath entrance we have the potential $V_{s e}$ which will be deduced from the parallel transport equations in section VII.

The ions will be accelerated in the sheath. If we assume now following the derivation in [3] that the parallel ion flux density remains constant within the very thin sheath, $n_{i} v_{i}=$ const, and for a moment that $T_{i}=0$, we can use ion energy conservation $\frac{1}{2} m_{i} v_{i}^{2}=-e V$ (no change of thermal energy) to obtain

$$
n_{i}(z)=n_{s e}\left(V_{s e} / V\right)^{1 / 2} .
$$

We use Eqns. 24 and 25 to transform 23 to

$$
\frac{\partial^{2} V}{\partial z}=-\frac{e}{\varepsilon_{0}} n_{s e}\left[\left(\frac{V_{s e}}{V}\right)^{1 / 2}-\exp \left(e\left(V-V_{s e}\right) / k T_{e}\right)\right] .
$$

Now we consider the region just inside the sheath where $\Delta V \equiv V_{s e}-V>0$ is small with respect to $V$ and expand the two terms on the RHS of Eqn. 26

$$
\begin{gathered}
\left(\frac{V_{s e}}{V}\right)^{1 / 2} \approx 1+\frac{1}{2} \frac{\Delta V}{V_{s e}}=1-\frac{1}{2} \frac{\Delta V}{\left|V_{s e}\right|} \\
\quad \exp \left(e\left(V-V_{s e}\right) / k T_{e}\right) \approx 1-\frac{e \Delta V}{k T_{e}}
\end{gathered}
$$


to get

$$
\frac{\partial^{2} \Delta V}{\partial z}=\frac{e \Delta V}{\varepsilon_{0}} n_{s e}\left(\frac{e}{k T_{e}}-\frac{\Delta V}{\left|V_{s e}\right|}\right) .
$$

From the condition that the electric potential in the Debye sheath has a monotonic distribution it follows that

$$
\begin{aligned}
\frac{e}{k T_{e}} & \geq \frac{\Delta V}{\left|V_{s e}\right|} \\
m_{i} v_{s e}^{2} & \geq k T_{e} \\
v_{s e} & \geq c_{s}
\end{aligned}
$$

where $c_{s}$ is the sound velocity and Eqn. 30 defines the Bohm criterion for the "plasma exit velocity". It will be supplemented with a condition $v_{s e} \leq c_{s}$ following from the calculation of the parallel velocity profile in the SOL as described in section VII to end with $v_{s e}=c_{s}$ as boundary conditions for the parallel flow at the sheath entrance (valid as long no drifts are considered, cf. section VIII).

Using this Bohm criterion we can describe the ion flux density to the target as the parallel flux density at the sheath entrance (se) (neglecting additional sources in the very thin sheath)

$$
\Gamma_{\text {target }}^{i}=n_{e s} c_{s}=\frac{1}{2} n(0) \sqrt{\frac{k\left(T_{i}+T_{e}\right)}{m_{i}}} .
$$

To preserve ambipolarity the ion flux (for an ion charge $\mathrm{Z}=1$ ) must balance the electron flux which is influenced by the sheath potential drop $V_{s}$. The electron distribution remains Maxwellian in the retarding electric field. Thus, the electron flux to the target reads

$$
\Gamma_{\text {target }}^{e}=\frac{1}{4} n_{e s} \bar{c}_{e}=\frac{1}{4} n_{e s} \exp \left(\frac{e V_{s}}{k T_{e}}\right) \sqrt{\frac{8 k T_{e}}{\pi m_{e}}} .
$$

Equating 31 and 32 yields

$$
\frac{e V_{s}}{k T_{e}}=0.5 \ln \left(2 \pi \frac{m_{e}}{m_{i}}\right)\left(1+\frac{T_{i}}{T_{e}}\right) .
$$

Typical values for the ratio given above are about 3. To quantify the total potential drop between stagnation plane and target surface one has to add the presheath potential drop deduced in the next section VII (cf. Eqn. 48). Emission of electrons from the surface reduces the electrostatic potential. In some cases it can even lead to a breakdown of the sheath. The most important effect is the emission of secondary electrons, but also reflected electrons, photon induced emission and thermal emission play a role. In particular, above certain temperatures thermal emission can dominate and is considered to be one reason for the formation of so called hot spots [18].

The ions gain energy in the sheath (at the expense of the electrons which are cooled because only the fast part of the electron population can leave the plasma while the slower ones are repelled by the sheath potential). The impact energy of ions to the target

$$
E_{\text {ion }}=2 k T_{i}+3 Z k T_{e}
$$

with $Z$ the charge of the ions, is significantly increased by the acceleration in the sheath, especially for highly charged impurity ions, leading to enhanced physical sputtering (cf. discussion in [2]).

The heat flux density of ions and electrons from the plasma onto the surface can be related to the particle flux densities leaving the plasma with the help of the so called "sheath transmission coefficients" defined as

$$
\gamma_{i, e}=\frac{q_{i, e}}{k T_{e} \Gamma_{\text {target }}}
$$

For electrons we get $\gamma_{e} \approx 2+3+0.5$ from the thermal, sheath and pre- sheath contribution. The ions don't have a Maxwellian distribution, if they had, then $\gamma_{i} \approx 2.5 T_{i} / T_{e}+0.5+0.5 T_{i} / T_{e}$. Numerical simulations allowing for non- Maxwellian ion distributions give somewhat smaller results $\gamma_{i} \approx 2-3$. The total sheath transmission coefficient is then around $\gamma=8$. The heat flux density to the target can be expressed as

$$
q_{\text {target }}=\gamma n_{e s} c_{s} k T_{e}=n_{e s} \sqrt{\frac{k\left(T_{i}+T_{e}\right)}{m_{i}}} k T_{e} .
$$

We finally can include the power flow deposited on the target upon recombination of the ion-electron pairs and possibly formation of molecules. This potential energy flow density can be expressed as

$$
q_{p}=n_{e s} c_{s} k \varepsilon_{p}=n_{e s} \sqrt{\frac{k\left(T_{i}+T_{e}\right)}{m_{i}}} \varepsilon_{p} .
$$

with the potential energy $\varepsilon_{p}$ composed of the ionization and dissociation energy of deuterium atoms and molecules, $\varepsilon_{p} \approx 16 \mathrm{eV}$.

The magnetic field $\vec{B}$ has no influence on the sheath description as long as the surfaces are orthogonal to $\vec{B}$. In practice, however, surfaces are tilted to spread the power onto the target. By enlarging the angle $\Psi$ between $\vec{B}$ and the normal to the surface the power flux density normal to the target can be reduced to 


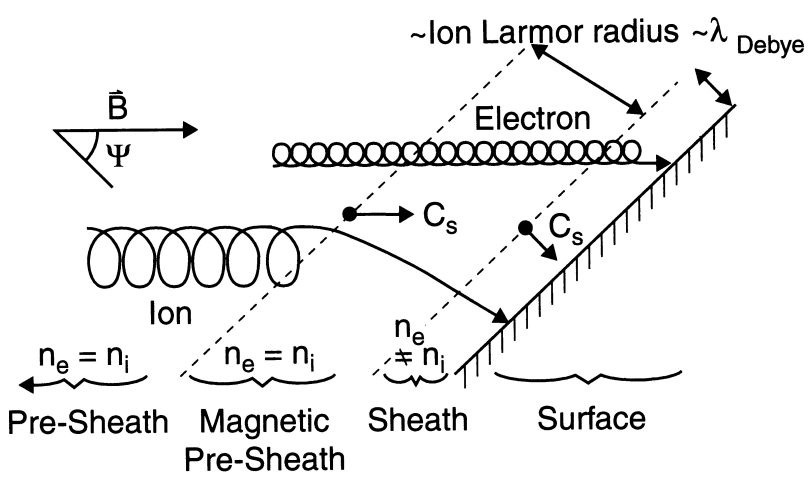

Figure 4: Near surface region for a target inclined to the magnetic field [13] (figure adapted from [3])

$$
q_{\text {dep }}=q_{\|} \cos \Psi .
$$

The parallel heat flux density in a fusion reactor can be in the order of $100-1000 \mathrm{MWm}^{-2}$ so that a shallow inclination of the targets with $\Psi$ up to $89^{\circ}$ is envisaged. For these small angles of incidence a new feature, the magnetic pre-sheath [13] shows up, which extends from the electrostatic Debye sheath discussed before about one ion larmor radius $\left(\sim 10^{-3} \mathrm{~m}\right)$ into the plasma. The reason for the formation of the magnetic pre- sheath is, that there is now an ExB drift of the ions perturbing the last gyro-orbits in front of the target, while the electrons will follow the magnetic field lines virtually all the way to the surface because of their much smaller gyro radius (cf. fig. 4). As a consequence, the Bohm criterion for the parallel flow now applies at the magnetic pre- sheath edge.

Equating the ion flux at the magnetic pre- sheath (mse) with the electron flux at the electrostatic sheath entrance (se), again assuming ambipolar flow and no sources in the pre-sheath and the Boltzmann relation for electrons)

$$
n_{m s e} c_{s} \cos \Psi=n_{s e} c_{s}=n_{m s e} \exp \left(e V_{m s e} /\left(k T_{e}\right)\right) c_{s}
$$

leads to

$$
\frac{e V_{m s e}}{k T_{e}}=\ln \cos \Psi
$$

The total potential drop in Debye $V_{D S}$ and magnetic pre-sheath $V_{m s e}$ together is independent on $\Psi$ to first order as found in numerical calculations [13], instead $\Psi$ determines the split between $V_{m s e}$ and $V_{D S}$. Thus, to first order, the sink action of the solid surface acting on the plasma, with regard to both particle and power flows, is unaffected by the change from a normal to an oblique target, as are the sheath voltage drop and the ion impact energy onto the target (Eqn. 34).

\section{PROPERTIES OF THE SIMPLE SOL}

Now we return back to the description of the parallel flow in the SOL. First we will describe the case of the simple SOL (cf. fig. 2a), which is also called the sheath-limited regime. The model assumptions are the following:

- The 1D fluid flow is isothermal, $T_{i}=$ const. and $T_{e}=$ const., and there are no heat sinks or sources inside the SOL. Thus, we don't need the energy transport equations.

- There are no particle sources from ionization inside the SOL, the only source is diffusive cross field transport.

- The target is a perfect plasma sink, there are no volume sinks as recombination.

- The ions and electrons are fully decoupled.

The source term for the continuity equation along $\mathrm{z}$ is given by radial particle transport into the SOL (no ionization inside the SOL)

$$
\frac{\partial}{\partial z}\left(n v_{\|}\right)=S_{p}=-\frac{\partial}{\partial x}\left(D_{\perp} \frac{\partial n}{\partial x}\right)=\frac{D_{\perp} n}{\lambda^{2}} .
$$

For the ion momentum balance, we again neglect friction with neutrals (CX losses and ionization) and represent the electric field with the help of the Boltzmann relation Eq. 24 yielding

$$
m_{i} n v_{\|} \frac{\partial v_{\|}}{\partial z}=-k\left(T_{e}+T_{i}\right) \frac{\partial n}{\partial z}+m_{i} v_{\|} S_{p}
$$

with $S_{p}$ as given by Eq. 41 .

Defining a parallel Mach number $M_{\|}=v_{\|} / c_{s}$ and using the definition of the (isothermal) ion sound velocity $c_{s}=\sqrt{\left(k\left(T_{e}+T_{i}\right) / m_{i}\right.}$ we can now use Eqs. 41 and 42 to deduce two coupled equations which describe the variation of the density and the Mach number along $\mathrm{z}$

$$
\begin{gathered}
\frac{\partial n}{\partial z}=-\frac{n D_{\perp}}{c_{s} \lambda^{2}} \frac{2 M_{\|}}{1-M_{\|}^{2}} \\
\frac{\partial M_{\|}}{\partial z}=\frac{D_{\perp}}{c_{s} \lambda^{2}} \frac{1+M_{\|}^{2}}{1-M_{\|}^{2}}
\end{gathered}
$$

The divergence of these two equations for $M_{\|}= \pm 1$ defines the boundary condition of the flow at the sheath 
entrance (the Bohm criterion as mentioned before). Combining Eqs. 43 and 44 we get

$$
\frac{\partial n}{\partial M_{\|}}=-n \frac{2 M_{\|}}{1+M_{\|}^{2}}
$$

which can be integrated analytically:

$$
\frac{n}{n_{0}}=\frac{1}{1+M_{\|}^{2}}
$$

with $n_{0}$ the density in the stagnation plane where $M_{\|}(z=0)=0$. Therefore, the density drops from the stagnation point to the sheath entrance to half its value. As we assumed no variation of the particle source originating from cross field transport into the SOL and $|\partial n / \partial x|=n / \lambda=$ const., the SOL thickness $\lambda$ reduces towards the target proportional to the density further aggravating the problem of the high target load as indicated before.

The equation describing the variation of the Mach number along z reads

$$
M_{\|}-2 \arctan M_{\|}=\left(\frac{\pi}{2}-1\right) \frac{z}{L}
$$

Finally, combining Eq. 46 and 24 we get an equation for the potential in the pre-sheath

$$
V(z)=-\frac{k T_{e}}{e} \ln \left(1+M_{\|}(z)^{2}\right)
$$

Therefore, at $M_{\|}=1$ the total pre-sheath drop is given by $V \approx-0.69 k T_{e} / e$.

Fig. 5 depicts the variation of the plasma density, the Mach number and the electric potential as given by Eqs. 46, 47 and 48, respectively, from the stagnation plane $\mathrm{z} / \mathrm{L}=0$ to the sheath entrance $\mathrm{z} / \mathrm{L}=1$.

For the situation of a simple SOL the power flux density is determined by the power flow into the SOL and the power decay length together with the perpendicular extension of the target (i.e. the poloidal extension in case of a toroidal limiter), which is small as is the density decay length discussed in section III. At the same time the temperature at the target surface remains high, unless both the power flow into the SOL and the temperature at the LCFS are reduced by power loss mechanisms inside the confined volume (cf. [11]).

\section{IMPACT OF DRIFTS ON THE SOL FLOW}

Next we discuss briefly the influence of drift effects on the parallel particle transport in the SOL which have been identified as the cause of significant poloidal asymmetries in tokamaks ([14], [15], [16], [17], cf. also discussion in [3], chapter 18, and references therein).

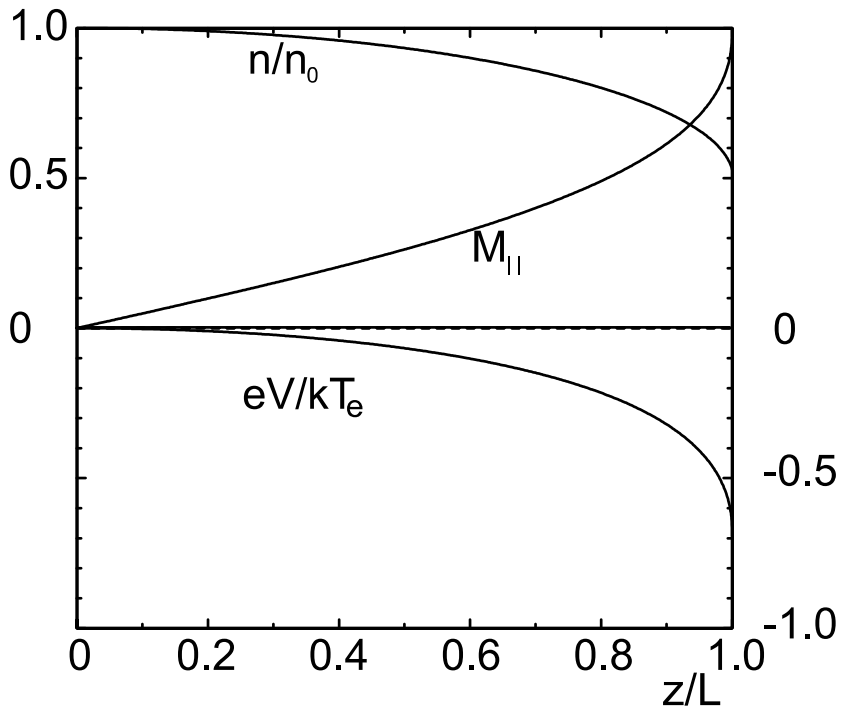

Figure 5: Variation of plasma density $\mathrm{n}$ normalized to $\mathrm{n}(0)$, parallel Mach number and normalized potential drop in the pre- sheath along the magnetic field from the stagnation plane $\mathrm{z} / \mathrm{L}=0$ to the sheath entrance $\mathrm{z} / \mathrm{L}=1$

The model for the parallel transport discussed before can be extended to include a perpendicular drift component caused by $E x B$ drift, diamagnetic drift and $\nabla B$ and curvature drift. We decompose the drift motion into a radial and a binormal component $\left(\vec{e}_{r} \perp \vec{e}_{\perp} \perp \vec{e}_{\|}\right)$which allows to express the poloidal velocity component $v_{\theta}$ as

$$
v_{\theta}=v_{\|} \sin \alpha+v_{\perp} \cos \alpha
$$

where $\alpha$ denotes the angle between the toroidal and poloidal magnetic field components as before $(\tan \alpha=$ $\left.\left.B_{\theta} / B_{\phi}\right)\right)$.

As we will continue to investigate transport along the field line, we have to project the resulting poloidal velocity onto the parallel direction (cf. fig. 6)

$$
\tilde{v}_{\|}=v_{\|}+\frac{1}{\tan \alpha} v_{\perp} .
$$

As a consequence Eqs. 43 and 44 are modified to

$$
\begin{gathered}
\frac{\partial n}{\partial z}=\frac{n D_{\perp}}{c_{s} \lambda^{2}} \frac{2 M_{\|}+M_{\perp} / \tan \alpha}{\left(M_{\|}+M_{\perp} / \tan \alpha\right)^{2}-1} \\
\frac{\partial M_{\|}}{\partial z}=\frac{D_{\perp}}{c_{s} \lambda^{2}} \frac{1+\left(M_{\|}+M_{\perp} / \tan \alpha\right)^{2}}{1-\left(M_{\|}+M_{\perp} / \tan \alpha\right)^{2}}
\end{gathered}
$$

where $M_{\perp}$ normalizes the perpendicular velocity to the sound speed. The boundary condition for the parallel Mach number at the sheath entrance reads

$$
M_{\|}(z= \pm L)= \pm 1-\frac{M_{\perp}}{\tan \alpha} .
$$




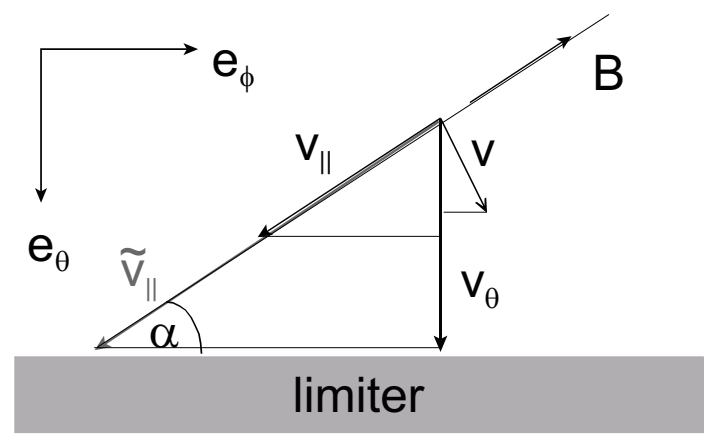

Figure 6: Projection of the perpendicular drift velocity onto the parallel direction.

Consequently, the flow towards both sides of the limiter or to the two divertor plates shown in Fig. 1 is asymmetric, resulting in an asymmetric density distribution along the field line and in poloidal direction. Eq. 46 is replaced by

$$
\frac{n}{n_{0}}=\frac{1}{1+M_{\|}\left(M_{\|}+M_{\perp} / \tan \alpha\right)} .
$$

Within the simple model discussed above (still under the assumptions of no ionizations in the SOL) Fig. 7 illustrates the influence of a perpendicular Mach number $M_{\perp}=0.05$ on the parallel Mach number along the field line from the electron drift side of the ALT- II limiter in TEXTOR (located $45^{\circ}$ below the outer midplane) to the ion drift side. The toroidal magnetic field and plasma current are anti- parallel under standard conditions in TEXTOR. In that case $M_{\|}>1$ on the ion drift side. The stagnation plane (as defined by $\mathrm{M}_{\|}=0$ ) is considerably shifted away from the geometrical symmetry plane (located at $z=0.5$; here we normalize $z$ to the total length of the flux tube $2 L$ ).

In poloidal divertor tokamaks drifts are thought to be the cause of strong asymmetries in the power load of inner and outer divertor target plates, so that both divertor zones are often in different divertor regimes. These divertor regimes are the topic of the following section.

\section{PROPERTIES OF THE COMPLEX DIVERTOR SOL}

At the end of section VII we have noted the problems of high power flux densities and high temperatures in limiter SOLs. While the high power flux densities

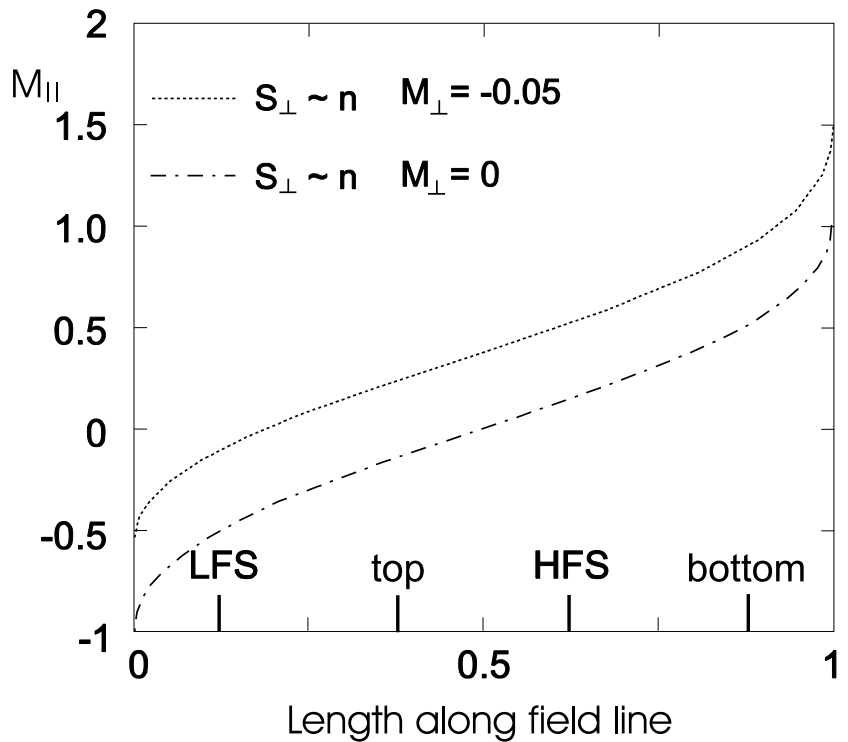

Figure 7: Influence of a perpendicular drift on the parallel Mach number $(\tan \alpha=0.1$, no ionization in the SOL)

lead to large temperatures of the surfaces of plasma facing components, high temperatures give rise to large energies of particles impinging onto the material surface (cf. Eqn. 34). Both effects will pose huge problems with the plasma- wall interaction in fusion devices (see [1] [2]). Therefore, the formation of a plasma regime with reduced power flow to and a cold plasma in front of the targets (conduction limited and high recycling regime) is an important issue in plasma edge physics.

The possibility to reduce the plasma temperature in front of the plasma facing components by establishing a temperature gradient along the magnetic field lines is closely related with the necessity to localize the ionization of neutrals close to the target. As it is depicted in fig. 2b) the parallel plasma flow is then only developing in the ionization (or recycling) region close to the target, most of the SOL is stagnant. In particular, there is no particle flux into the SOL from the confined volume. In practise, such a situation cannot be reached to a full extend because there will be also interaction of the plasma with the main chamber walls, leading to ionization sources remote from the divertor target or limiter inside the SOL or even sources in the confined plasma, if the neutrals recycling at the main chamber walls penetrate across the LCFS. Nevertheless, the particle flux from the core and into the SOL remote from the targets may be rather small with respect to the recycling flux at the targets. While the particle sources are close to the targets, the heat fluxes remain in the core of the confined plasma. Therefore, there is still the power flow out of the confined volume into the SOL and along the magnetic field towards the targets which constitute the heat sink. Without particle flow this heat 


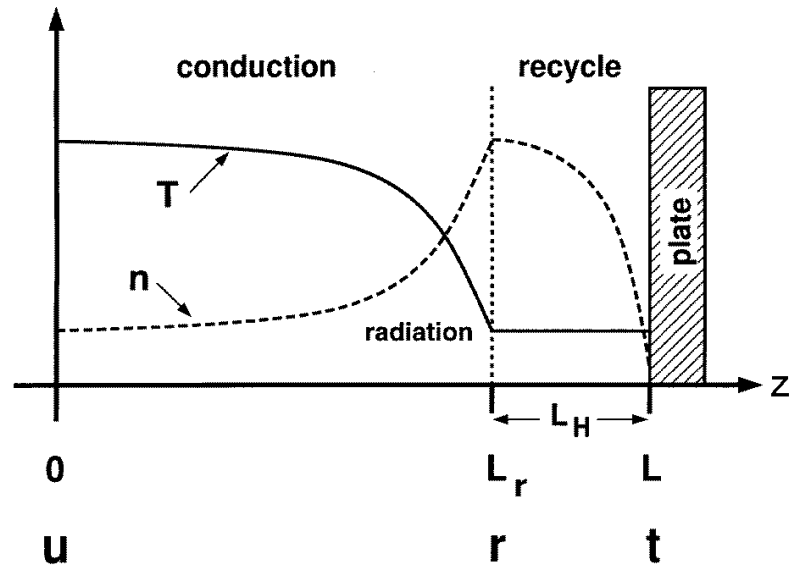

Figure 8: Schematic illustration of the two point model (figure adapted from [6])

flux cannot be convective but will be conductive and a temperature gradient must develop.

We can quantify the effects in the complex SOL of a divertor using a simple analytical model (the so called "two point model", cf. [3], chapters 4 and 5, and $[6])$. For simplicity we assume equal ion and electron temperatures, $T_{i}=T_{e}$. Fig. 8 illustrates the typical plasma profiles along the magnetic field for such a situation. We have two different regions, the conduction region consisting of most of the SOL where there are no sources or sinks, and the recycling region where we have the strong ionization sources and possibly a sink for energy and momentum. The two point model relates the conditions upstream of the target (position $\mathrm{u}$ ) to those at the target (position $t$ ) in the case where the fraction $f_{\text {cond }}$ of the power is conducted along the parallel temperature gradient as

$$
q_{\|, \text {cond }}=f_{\text {cond }} P_{S O L} / A_{q \|}=-\kappa_{0} T^{5 / 2} d T / d z,
$$

where $\kappa_{0}$ is taken for electrons because of their higher heat conductivity (cf. Eqns. 20 and 21), $P_{S O L}$ is the power flow into the SOL and $A_{q \|}$ the total crosssectional area of the SOL for power flow perpendicular to $\vec{B}$ (all power enters the SOL upstream of the target). We include possible volumetric power sinks characterized by the factor $f_{\text {loss }}>0$ in the balance between power flux into the SOL and power flux at the sheath entrance as

$$
\left(1-f_{\text {loss }}\right) P_{S O L} / A_{q \|}=\gamma n_{t} c_{s} k T_{t}
$$

We further introduce a factor $f_{\text {fric }}<1$ into the pressure balance to take pressure losses because of momentum sinks and friction into account

$$
n_{u} T_{u} f_{\text {fric }}=2 n_{t} T_{t}
$$

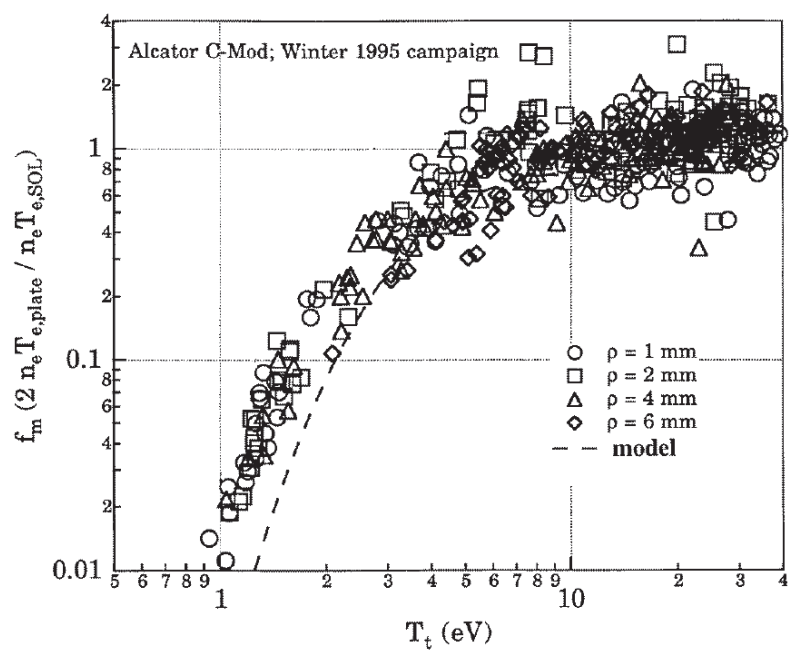

Figure 9: Pressure loss factor from ohmic discharges in C-Mod as a function of the electron temperature at the target $T_{t}$ and factor calculated from Eqn. 59 (figure adapted from [6]).

Momentum losses induced by CX processes between ions and neutrals in a high density divertor play a major role in in poloidal divertors and occur for very low temperatures below $7 \mathrm{eV}$, and we will come back later to an assessment of the factor $f_{\text {fric }}$. On the other hand, in the island divertor of helical devices detachment processes at higher temperatures have been observed, which have been related to friction losses caused by counter streaming flux tubes in the complicated $3 \mathrm{D}$ geometry of such an island divertor [30] [8]. An expression for $f_{\text {fric, } C X}$ has been been given in [6] following an 1D analytical solution for the momentum balance in [31]. Here it is assumed that the temperature in the recycling region shown in fig. 8 is constant and the momentum loss reduces the density only. The density ratio between the entrance of the recycle region $(r)$ and the target is given as

$$
\frac{n_{r}}{n_{t}}=\left(\frac{\alpha+1}{\alpha}\right)^{(\alpha+1) / 2}
$$

where the coefficient $\alpha$ is linked to the rate coefficients for ionization and charge exchange (cf. section IV) as $\left.\alpha \equiv<\sigma v\rangle_{i} /\left(\langle\sigma v\rangle_{C X}+<\sigma v\right\rangle_{i}\right)$. Using the pressure balance equation 57 we obtain

$$
f_{\text {fric }}=2\left(\frac{\alpha}{\alpha+1}\right)^{(\alpha+1) / 2}
$$

This Equation fairly well describes the experimentally determined pressure drop in the high density Alcator - CMOD tokamak [6] as shown in fig. 9. 
Returning back to the two point model we now integrate Eqn. 55 over the distance $L$ between the upstream region $u$ and the target region $t$ to obtain

$$
T_{u}^{7 / 2}=T_{t}^{7 / 2}-\frac{7}{2} \frac{P_{S O L} L}{A_{q \|} \kappa_{0}} f_{\text {cond }} .
$$

Because $T_{t}^{7 / 2} \ll T_{u}^{7 / 2}$ as soon as a temperature gradient exists the upstream temperature is given as

$$
T_{u}=\left(\frac{7}{2} \frac{P_{S O L} L}{A_{q \|} \kappa_{0}} f_{\text {cond }}\right)^{2 / 7}
$$

showing a very weak dependence on all parameters.

If we now take $n_{u}$ and $P_{S O L} / A_{q \|}$ as given, we can derive from Eqs. 56, 57 and 61 an expression for the temperature at the target

$$
T_{t}=\frac{m_{i}}{2 e} \frac{4\left(P_{S O L} / A_{q \|}\right)^{2}\left(\frac{7}{2} \frac{P_{S O L} L}{A_{q||} \kappa_{0}}\right)^{-4 / 7}}{\gamma^{2} e^{2} n_{u}^{2}} \cdot \frac{\left(1-f_{\text {loss }}\right)^{2}}{f_{\text {fric }}^{2} f_{\text {cond }}^{4 / 7}} .
$$

Correspondingly we get for the density $n_{t}$ at the target

$$
n_{t}=\frac{n_{u}^{3}}{\left(P_{S O L} / A_{q \|}\right)^{2}}\left(\frac{7}{2} \frac{P_{S O L} L}{A_{q \|} \kappa_{0}}\right)^{6 / 7} \frac{\gamma e^{2}}{2 m_{i}} \cdot \frac{f_{\text {fric }}^{3} f_{\text {cond }}^{6 / 7}}{\left(1-f_{\text {loss }}\right)^{2}}
$$

High upstream densities $n_{u}$ are very efficient to realize a cold and dense high recycling divertor with high $n_{t}$ and low $T_{t}$. We further note that the plasma conditions at the target depend very sensitively on the loss parameters $f_{\text {loss }}, f_{\text {cond }}$ and $f_{\text {fric }}$. A consistent picture of these parameters can only be obtained from sophisticated modelling (cf. the discussion in [5]).

Nevertheless, we will give some further considerations on the balance between convective and conductive heat flux density, which determines the desired temperature drop along the field lines.

As a first step we can deduce the ratio between upstream and target temperature from Eqn. 61 and 62 which scales as

$$
\frac{T_{u}}{T_{t}} \propto n_{u}\left(\frac{7}{2} \frac{P_{S O L} L}{A_{q \|} \kappa_{0}}\right)^{6 / 7} \frac{f_{\text {fric }}^{2} f_{\text {cond }}^{6 / 7}}{\left(1-f_{\text {loss }}\right)^{2}} .
$$

Naturally, the existence of conductive heat transport $\left(f_{\text {cond }}>0\right)$ and the resulting temperature ratio is directly linked, and any contribution heat convective heat transport will reduce $T_{u} / T_{t}$. Volumetric power losses close to the target strongly drive the temperature drop but momentum losses impede it. But what is now the ratio between convective and conductive heat flux and how to control it? Why we observe high recycling regimes in divertor configuration but not in limiter machines? This difference is of course strongly linked to the possibility to retain the ionization sources inside of the SOL. To quantify this statement we have integrated the balance equation for the total energy (sum of Eqns. 20 and 21) retaining both conductive and convective heat flux densities. We used the power flux density as well as the particle flux density to the target as a boundary condition. The convective heat flux is driven by the particle sources inside the confined plasma $f_{S O L} \Gamma_{0}$ where we assume complete recycling at the target, $\Gamma_{t, \|}=\Gamma_{0}$. We obtain

$$
\begin{aligned}
\frac{\partial q_{\|}}{\partial z} & =\frac{q_{\|, t}}{L} \\
& =\frac{\partial}{\partial z} q_{\|, \text {cond }}+\frac{\partial}{\partial z} q_{\|, \text {conv }} .
\end{aligned}
$$

Here we have the parallel heat flux to the target given by the power $P_{S O L}$ entering the SOL all along the connection length $L$, the cross section of the heat flux channel $A_{q \|}=4 p i R \lambda_{q \|} a /\left(q_{a} R\right)$ and the energy loss by ionization and excitation $E_{i}=30 \mathrm{eV}$ [4] given as

$$
q_{\|, t}=\frac{P_{S O L}}{A_{q \|}}-f_{S O L} \Gamma_{0} E_{i}=\gamma \Gamma_{t, \|} k T_{t}
$$

and the rate of change of the conductive and convective heat flux density given as

$$
\begin{aligned}
\frac{\partial}{\partial z} q_{\|, \text {cond }} & =-\kappa_{e 0}\left[T^{5 / 2} \frac{\partial^{2} T}{\partial z^{2}}+\frac{5}{2} T^{3 / 2}\left(\frac{\partial T}{\partial z}\right)^{2}\right] \\
\frac{\partial}{\partial z} q_{\|, \text {conv }} & =\left(1-f_{S O L}\right) k \frac{\Gamma_{0}}{L}\left(5 T+z \frac{\partial T}{\partial z}\right) \cdot
\end{aligned}
$$

The second order differential equation 65 is solved for $T$ numerically after transformation into two first order equations

$$
\begin{aligned}
\frac{\partial T}{\partial z}= & \tilde{T} \\
\frac{\partial \tilde{T}}{\partial z}= & -\left(\kappa_{e 0} L\right)^{-1} T^{-5 / 2}\left[q_{\|, t}-\frac{\Gamma_{0}}{\left(1-f_{S O L}\right) k}(5 T+z \tilde{T})\right] \\
& -\frac{5}{2} \tilde{T}^{2} T^{-1}
\end{aligned}
$$

The boundary conditions are

$$
\left.\frac{\partial T}{\partial z}\right|_{z=0}=0 \text { and }\left.T\right|_{z=L}=T_{t}
$$

The target temperature is defined by the inferred heat flux density to the target Eqn. 66. The target density follows from the particle flux to the target, and target temperature and the upstream densities and temperatures are determined by the pressure balance Eqn. 57 where no momentum losses are considered. 
We use typical parameters for the limiter tokamak TEXTOR, major radius $R=1.75 \mathrm{~m}$, minor radius $a=0.46 m$, connection length $L=20 \mathrm{~m}$, edge safety factor $q_{a}=3$, power entering into the SOL $P_{S O L}=1 M W$, power decay length $\lambda_{q \|}=0.02 m$ and vary then the particle flux density onto the target. We use the fraction of ionizations inside the SOL $f_{S O L}$ as a parameter. The results are shown in fig. 10.

We scanned the collisionality $\nu_{*}=L / \lambda_{e e}$ as the ratio between the connection length and the mean free path of electrons (or ions) in a wide range and calculated the ratio of upstream and target temperature $T_{u} / T_{t}$, absolute values of upstream and target temperatures, $T_{u}$ and $T_{t}$ as well as fraction of power lost on ionization and excitation of neutrals in the SOL $f_{\text {loss }}$. The calculation is stopped as soon as the target temperature approaches a value of $7 \mathrm{eV}$ because at these temperatures momentum dissipation by CX processes will become significant. We clearly see that the fraction of ionizations in the SOL has to be 0.6 and higher to allow for a significant temperature drop along the magnetic field. Such large neutral screening is, however, inaccessible in a limiter device because neutrals recycling at the limiter penetrate into the confined zone even at highest densities when the temperature falls below the ionization threshold for hydrogen.

Our result is consistent with [32] where an improved two point model including both convective and conductive heat transport as well as a realistic description of the neutrals and the resulting particle sources had been developed for a limiter SOL and compared to full numerical simulations with a fluid code coupled to a Monte-Carlo code for the neutrals (both codes use full $3 \mathrm{D}$ geometry). Here the numerical code shows that at maximum $50 \%$ of the neutrals can be ionized in the SOL of TEXTOR. As a consequence, the ratio $T_{u} / T_{t}$ saturates at high collisionality. Higher neutral screening is only possible in a divertor tokamak where the targets are positioned remote from the confined volume inside. Closed divertor configurations which suppress leakage of neutrals out of the divertor chamber are best in this respect (cf. also the discussion in [5]).

As we see from fig. 10 under conditions of good neutral screening from the divertor we quickly approach a situation, where the temperatures in the recycling region are small enough to allow for large momentum losses, leading to a detached divertor state. Then the particle and power flux to the divertor plate is strongly reduced. Such a regime is envisaged for fusion devices. However, stability of a detached divertor is an issue as we can expect from the complicated and non-linear interplay of the various loss channels. Also the need for additional losses by radiation from impurities is a subject of current research. In present days experiments
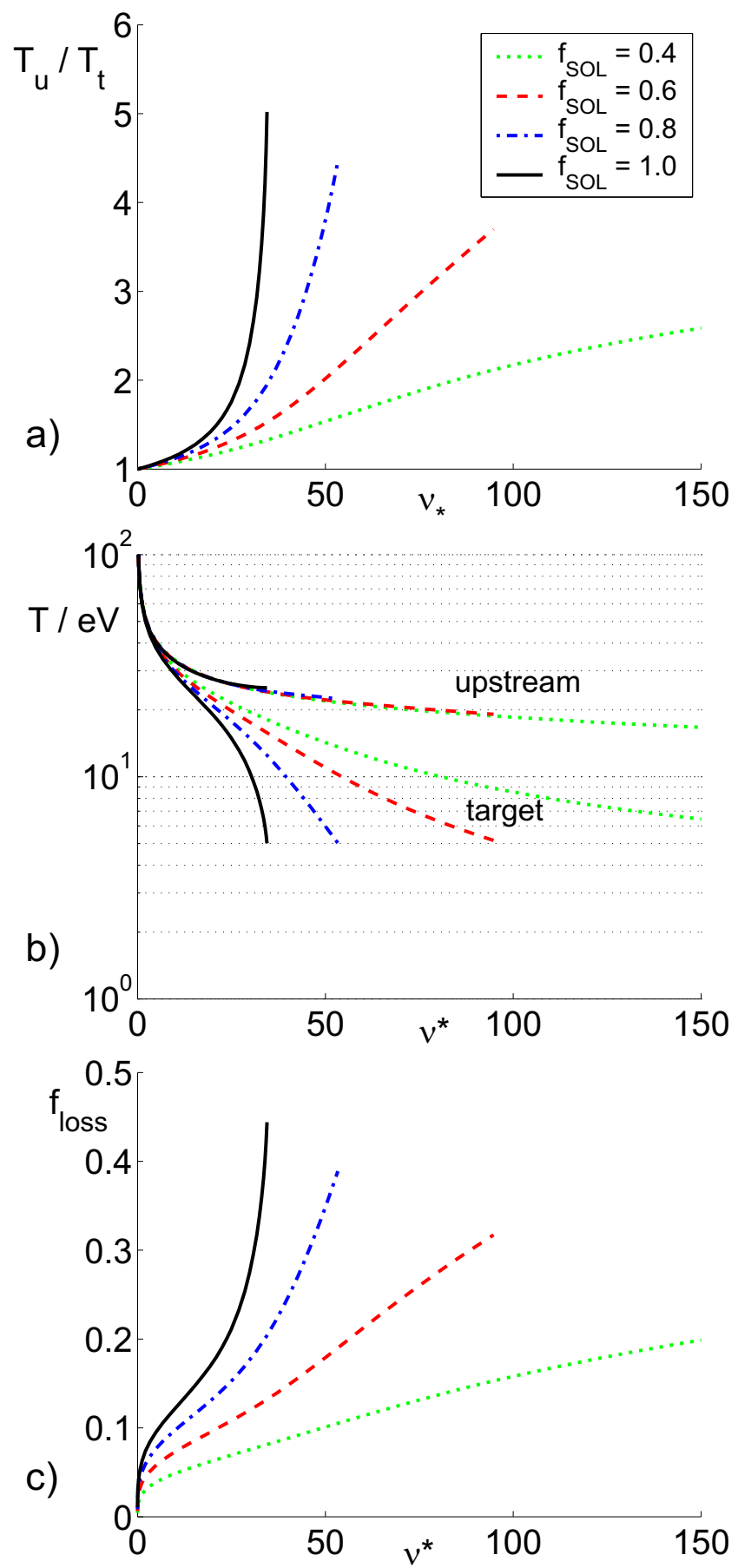

Figure 10: Variation of SOL parameters as a function of the upstream collisionality for typical TEXTOR conditions using the fraction of ionizations inside the SOL as parameter: a) ratio of upstream and target temperature, b) absolute values of upstream and target temperatures, b) fraction of power lost on ionization and excitation of neutrals in the SOL 
the high heat load areas of the divertors are mostly made of carbon, an effective intrinsic radiator at the low temperatures close to detachment (see the extensive discussion in [5]).

\section{ACKNOWLEDGMENTS}

I wish to thank Michael Lehnen for Figs. 6 and 7 and for the model to calculate the parallel heat flux in the TEXTOR SOL.

\section{REFERENCES}

1. U. Samm, "Plasma- wall interaction in magnetically confined fusion plasmas", these proceedings.

2. A. Kirschner, "Erosion and deposition mechanisms in fusion plasmas", these proceedings.

3. P.C. Stangeby, "The Plasma Boundary of Magnetic Fusion Devices", Plasma Physics Series, IoP Publishing Ltd, Bristol, UK (2000).

4. A.V. Nedospasov and M.Z. Tokar', "Wall plasma in tokamaks", in Reviews of plasma physics, B.B. Kadomtsev (ed.), Vol. 18, Consultants Bureau, New York (1993).

5. R. Schneider, "Plasma edge physics for tokamkas", Laborbericht IPP Garching, IPP 12/1, February 2001

6. C.S. Pitcher and P.C. Stangeby, Plasma Phys. Control. Fusion 39 (1997) 779-930.

7. M.Z.. Tokar, "Stochastic boundary plasmas", these proceedings.

8. R. König et al., Plasma Phys. Control. Fusion 44 (2002), 2365.

9. D. Reiter, "Recycling and transport of neutrals", these proceedings.

10. D. Reiter, G.H. Wolf and H. Kever, Nucl. Fusion 30 (10) 2141 (1990).

11. M.Z. Tokar, "Impurity transport and radiation at the edge", these proceedings.

12. S.I. Braginskii, "Transport processes in a Plasma", in Reviews of Plasma Phyiscs, M. Leontovich (ed.), Consultants Bureau, New York (1965), 205.

13. R. Chodura, "Plasma flow in the Sheath and Presheath of a Scrape-off Layer", "Physics of PlasmaWall Interaction in Controlled Fusion Devices", ed D.E. Post and R. Behrisch, Plenum Press, New York (1986), p. 99.
14. H. Gerhauser and H.A. Claassen, J. Nucl. Mater. 176-177, 721 (1990).

15. M. Baelmans, D. Reiter and R.R. Weynants, Contrib. Plasma Phys., 36117 (1996).

16. M. Lehnen, M. Brix, U. Samm, B. Schweer, B. Unterberg and the TEXTOR- team, Nucl. Fusion 43 168. (2003).

17. M. Lehnen, M. Brix, H. Gerhauser, B. Schweer and R. Zagorski, J. Nucl. Mater. 290-293 (3) 663 (2001).

18. V. Philipps et al., Nucl. Fusion 33 (6), 953 (1993).

19. P. Franzen and E. Vietzke, J. Vac. Sci. Technol. A12 820 (1994).

20. A. Pospieszczyk et al., J. Nucl. Mater. 266-269 138 (1999).

21. M.F.A. Harrison in "Applied Atomic Collision Physics", Vol.2, Academic Press 1984, Eds. C.F. Barmett and M.F.A. Harrison (1984).

22. J.D. Hey et al., Contrib. Plasma Phys. 36583 (1996).

23. A. Pospieszczyk and Ph. Mertens, J. Nucl. Mater. 266-269 884 (1999).

24. D. Reiter, J. Nucl. Mater. 196-198 80 (1992).

25. B. Lehnert, Nucl. Fusion 8173 (1968).

26. H.P. Summers and M. von Hellermann, "Atomic and molecular data exploitation for spectroscopic diagnostics of fusion plasmas", in "Atomic and plasma- material processes in controlled thermonuclear fusion", Edt. by R.K Janev and H.W. Darwin, Elsevier, Amsterdam (1993), 87., http://adas.phys.strath.ac.uk/

27. H.R. Griem, "Plasma Spectroscopy", Mc GrawHill Book Company, New York (1964)

28. H.P. Summers and R.W.P. McWhirter, J. Phys. B 12 (1979), 2387.

29. R.W.P. Mc Whirter and H.P. Summers, "Atomic Radiation from Low Density Plasma" in Applied Atomic Collision Physics, Vol. 2, ed. C.F. Barnett and M.F.A. Harrison, Academic Press, Orlando (1984).

30. P. Grigull et al, Plasma Phys. Control. Fusion 43 (2001), A175.

31. S.A. Self and H.N. Ewald, Phys. Fluids 9 (1966), 2486 .

32. M.Z. Tokar', M. Kobayashi and Y. Feng, Phys. of Plasmas 11 2004, 4610. 